\title{
REFLEXIONES SOBRE LA CONTRIBUCIÓN DE LA TUTORÍA AL FUTURO DEL APRENDIZAJE
}

\author{
Richard Elmore
}

A finales de octubre y principios de noviembre ayudé a organizar un taller para promotores de tutoría en el estado de Guanajuato, México. El propósito del taller fue involucrar a los adultos promotores de tutoría en los primeros esfuerzos por generar de manera más explícita normas y prácticas sobre el papel tanto de adultos como de gente joven en el trabajo de tutoría. Hasta ahora la tutoría se ha difundido de viva voz, en demostraciones individuales, cara a cara y en eventuales sesiones de desarrollo profesional, pero sin la intención explícita de codificar la práctica. El taller de Guanajuato se concibió expresamente para ver si una formulación más explícita de la práctica tutora ayudaría a desarrollar la nueva manera de enseñar y aprender así como acelerar su difusión.

No es nada seguro que si se logra desarrollar normas más explícitas, mejorará la práctica de la tutoría y su difusión será más rápida. La práctica de la tutoría no se ha codificado rigurosamente hasta ahora, pero eso no parece haber impedido ni su desarrollo ni su difusión, como trataré de explicarlo más adelante. Siempre hay peligro que el exceso de codificación persuada a los recién llegados que basta seguir ciertas rutinas y comportamientos para adquirir maestría, con lo que se perdería la esencia de la tutoría, sus ideas fuerza, su teoría y la manera cómo se vive en la práctica. Con todo hay algunas razones que llevan a pensar que experimentar con normas y procedimientos más claros ayudará a mejorar la práctica. La principal razón es que la práctica de la tutoría está extendiéndose, más allá del alcance del grupo que la promueve en México, hacia Latinoamérica y, al menos potencialmente, a los Estados Unidos. Al observar los intercambios entre quienes dominan el arte de la tutoría y quienes la experimentan por primera vez, tanto en Chile como en los Estados Unidos, veo el profundo impacto que les produce, así como la satisfacción y el deseo de hacerse competentes en la metodología, pero necesitarían contar con guías más claras para adoptar las normas y principios que sustentan la práctica. Es posible que un conjunto de normas más explícitas ayude a sostener la difusión de la práctica a otros lugares, donde el modo tradicional de difusión, cara a cara, será menos viable, particularmente cuando el número de adesiones al modelo rebase con mucho la capacidad del núcleo promotor en México. Pero es también posible 
que el modelo de difusión de la práctica, cara a cara, sea de tal manera integral a la cultura de la tutoría que resulte imposible implementarla sin recibir capacitación de quienes ya la dominan. Esto queda como pregunta abierta.

El taller de Guanajuato tuvo éxito, pero relativo, diría yo, en el limitado propósito de producir formulaciones iniciales para normar la práctica. Los participantes, con diversos grados de experiencia y dominio de la técnica, contribuyeron gustosos al esfuerzo, aunque podrían no estar totalmente convencidos del provecho que traería. Los coordinadores procedieron con la magia propia de la tutoría, llevando al límite a los participantes para extraer sus ideas, sin adelantarse a dar respuestas, como es la práctica tutora. Al final, con algunos ajustes y mejor edición se logró una primera descripción bastante aceptable del desempeño progresivo de tutores y aprendices desde el nivel de novicio hasta el de quien se maneja ya como un experto en tutoría. (Se pueden consultar estas rúbricas al final en los Apédices A y B) Una idea central de este ejercicio fue que la práctica no se domina sino incrementalmente. Uno no adquiere una práctica compleja simplemente adoptando y ejecutando una serie de reglas. La práctica se adquiere al pasar por etapas en las que se va dominando y entendiendo mejor el proceso; generalmente ayudado por alguien con mayor experiencia. El taller, en otras palabras, se diseñó tratando de ser congruentes con los principios y la práctica de la tutoría. Además de dirigir el taller, en Guanajuato visité una de las escuelas donde una alumna me dio tutoría; también pude intercambiar puntos de vista con coordinadores y funcionarios estatales. La experiencia fue importante para conocer una vez más la variedad de entornos en los que opera la tutoría. Mi experiencia, que está lejos de ser completa, se ha ido incrementando con visitas a centros donde se practica la tutoría en Zacatecas, Tijuana, Mexicali y ahora Guanajuato. Espero seguir teniendo la oportunidad de visitar prácticas de tutoría en otras partes. También he podido observar el desarrollo de una muy promisoria sesión de capacitación por parte de promotores mexicanos a colegas chilenos que reciben apoyo de fundaciones para promover una red de difusión de la tutoría en Chile.

\section{De la práctica a la teoría: el gozo de aprender}

Como parte de mi trabajo actual de consultor y docente en línea, aunque como desfío muy personal, emprendí el estudio de dos temas complejos: (I) la neurosciencia del conocimiento; literalmente, cómo opera la mente cuando asimila y procesa conocimientos nuevos y como integra y entiende realidades complejas, y (2) la relación entre el diseño de un espacio y sus ocupantes, la manera como lo perciben y lo que en ese espacio se aprende. Pienso que de estos dos temas depende el futuro del aprendizaje. Además de estudiar estos estos temas, actúo como consultor de empresas que diseñan espacios propicios para el aprendizaje y sistematizo los 
resultados para uso de quienes decidirán políticas educativas en contextos sociales más amplios.

La tutoría ocupa un nicho muy especial entre los casos ejemplares que habrán de guiar el futuro del aprendizaje; especial por la importancia de lo que enseña y el poder de lo que logra. Es una práctica cuyo diseño lleva a desarrollar una teoría del aprendizaje cada vez más radical y compleja, aunque sostenida en todo momento por la práctica misma. La práctica es relativamente simple, la teoría lleva a entender con mayor claridad y precisión la compejidad del proceso de aprendizaje en jóvenes y adultos. En este sentido la tutoría invierte la relación habitual en las ciencias sociales entre teoría y práctica, y genera una cultura centrada en lo que describiría como "la búsqueda deliberada de sorpresas". La práctica se desarrolla a través de preguntas no de respuestas. La esencia de la relación tutora es dar el mayor control posible al aprendiz sobre lo que quiere estudiar y organizar el diálogo sobre el descubrimiento que él debe hacer, ayudado por su el tutor, para no sólo entender lo que escogió, sino cómo y por qué se estructura lo que ahora entiende. La tutoría lleva a razonar y descubrir, no a encontrar pronto la respuesta que el maestro espera. En mi experiencia al recibir tutoría y al observar la que otros reciben, cuando es buena tutoría, me ha sorprendido siempre la tensión que se genera y la constante apertura a la sorpresa. El aprendiz va siendo guiado en una búsqueda en la que no está seguro cuál será finalmente el resultado. El tutor, independientemente de las veces que haya dado tutoría sobre el mismo tema, espera siempre sorpresas en la manera particular como su aprendiz aborda y asimila el tema. Las preguntas que se hacen en la tutoría no son para inducir respuestas "correctas" sino para profundizar la compensión del tema tanto por parte del aprendiz como del mismo tutor. En este contexto lo inesperado es lo más esperado, no algo aberrante que se debe evitar. Después diré cómo esta búsqueda deliberada de sorpresas encarna un poderoso atisbo de la neurociencia. Por el momento baste decir que esta práctica ilustra una importante teoría del aprendizaje que surge de la neurociencia.

Otro aspecto importante en el que la tutoría se diferencia de prácticas de aprendizaje novedosas es que no depende en absoluto del lugar en el que se ejerce. En estos días pienso largamente sobre el impacto que los entornos físicos pueden tener en los aprendizajes. Urge conocer esto porque (a) las escuelas, con contadas excepciones, son entornos muy dañinos para el aprendizaje de los chicos, no digamos ya para pasar en ellas 16,000 horas de sus vidas, y (b) la escolaridad es una industria sumamente intensiva en capital que consume grandes cantidades de dinero público, repitiendo prácticas de aprendizaje profundamente disfuncionales y en entornos físicos profundamente inadecuados. Ya hay un basto cuerpo de doctrina en la neurociencia sobre el efecto del espacio físico en el aprendizaje, y sobre el tema más particular de cómo afectan las condiciones del entorno a quienes en él aprenden -información 
que prácticamente no consideran los educadores y administradores que deciden los principales montos y destinos de las inversiones. Los casos que estudio, en los que se busca explícitamente que el entorno físico favorezca el aprendizaje, tienen todos en común estar diseñados siguiendo teorías de aprendizaje bien definidas, algunas muy discutibles, pero a las que deliberadamente se acomodan los elementos del diseño; en ocasiones de manera muy tentativa ( y no siempre con éxito), aunque en todo momento lo que se busca es que el entorno favorezca el empeño del aprendiz.

Lo que me fascina de la tutoría es su total indiferencia a los lugares. He visto dar tutoría en lugares sumamente pobres, donde solo hay un techo miserable, deficiente luz y los estudiantes trabajan fuera del salón sobre piso de tierra. He visto dar tutoría en escuelas que evidentemente hace poco han sido renovadas, pero que simplemente siguen siendo versiones nuevas, más limpias, del modelo tradicional. He visto dar tutoría en escuelas que cuentan con acceso a la red digital y en escuelas donde no hay computadoras. He visto que se da tutoría en escuelas de donde es prudente salir a tiempo para evitar asaltos y daños físicos; pero también en comunidades tranquilas donde no asecha ningún peligro. Me preguntarán si tengo propuestas para diseñar espacios físicos que favorezcan la práctica de la tutoría y respondo que sí; pero tengo que reconocer que el poder de esa práctica no depende del espacio que la acoge, su accoutrement, sino del poderoso y radical estilo personal de aprendizaje, así como de la teoría que la sustenta.

En contraste, otro caso ejemplar que conozco es el de Hi Tech High, ahora un conjunto de escuelas en San Diego, que experimentan una teoría divergente de aprendizaje, a partir de contenidos integrales que se trabajan a base de proyectos. La planta física refleja y refuerza de manera explícita la teoría pedagógica, aunque es claro que tanto la teoría de aprendizaje como las prácticas que ésta implica empiezan ya a a rebasar las posibilidades de la planta física. Otro caso es NuVu en Cambridge, Massachusetts, que se diseñó explícitamente siguiendo el modelo de aprendizaje en escuelas de diseño arquitectónico, en las que para enfrentar un problema de la vida real se discute en común, se proponen prototipos físicos o virtuales, se objeta, se rediseña y finalmente se logra un producto que satisface al conjunto. Los adultos en NuVu son arquitectos y diseñadores apasionados por el aprendizaje. NuVu cuenta con abundantes instrumentos y materiales para diseñar y construír, además de ser un entorno desbordante de energía a cualquier hora.

Cuando pienso en la relación del entorno físico y el aprendizaje, descubro la importante lección que nos da la tutoría: podemos saber mucho y muy valioso sobre la relación entre planta física y aprendizaje, pero sin una poderosa teoría del aprendizaje, todo ese conocimiento será prácticamente inútil para diseñar (aunque a la tutoría la tiene sin cuidado) el entorno físico. Lo que la tutoría me enseña es que las mejores teorias sobre el aprendizaje no suponen necesariamente inversiones 
considerables en planta física, y que cuando todas esas mejoras no se sustentan en buenas teorías, es casi seguro que se pierden. En el área metropolitana de Boston, las dos preparatorias más recientes, monumentales, costaron cientos de millones de dólares, pero se diseñaron teniendo en cuenta una teoría del aprendizaje (si se le quiere dar tan respetable nombre) que apareció por I 870 y continúa dominando con aliento de muerte, hasta el día de hoy, el trabajo educativo en las secundarias de los Estados Unidos.

Finalmente, como lo he dicho en muchas ocasiones, la tutoría nos ofrece una poderosa alternativa para imaginar y llevar a la práctica nuevas maneras de aprender en nuestra sociedad. Mi primera reacción después de visitar Zacatecas fue decir que la importancia de la tutoría estaba en mostrarnos cómo organizar la transformación del aprendizaje con el esquema de los movimientos sociales más que con el de las burocracias jerárquicas. Desde entonces he estado reflexionando sobre lo que hice en los últimos quince años buscando la manera de involucrar a los maestros en lo que podríamos llamar "mejoramento de la práctica profesional" -es decir, atenta observación y análisis de la práctica docente, así como reflexión y diagnóstico sobre la gestión escolar. Mi triste conclusión es que las prácticas de mejora profesional tienen poco o nulo impacto en entornos que responden a políticas educativas muy institucionalizadas. Otra manera de decir esto es que las decisiones de política educativa han fallado en el intento de lograr cambios duraderos y a gran escala, ya no digamos para transformar los aprendizajes escolares. Las políticas que deciden las jerarquías son para generar y distribuír dignidad y poder entre los grupos subordinados, no para iniciar cambios divergentes o cambios que desestabilicen el sistema. Gabriel Cámara lo dijo bien durante nuestra visita a San Diego y ciudades de la frontera con México. Comentando en una de las sesiones en la que la discusión llegó, como es frecuente, al tema de lo difícil que resulta introducir una nueva práctica en la rígida estructura institucional, dijo: "Pienso que dedicamos demasiado tiempo y energía tratando de enderezar lo que está torcido; mejor empezar bien desde el principio".

Estoy convencido que el sobreinstitucionalizado entorno en el que tiene lugar la educación pública continuará vigente por largo tiempo, independientemente de qué tan capaz sea para promover el cambio y su indiferencia hacia transformaciones y ventajas para aprender que están teniendo lugar en la sociedad más amplia. El entorno institucional actual es demasiado grande y está demasiado comprometido con poderosos intereses políticos como para reconocer, mucho menos tratar de enmendar, sus profundas patologías y distorsiones. Al mismo tiempo hay que reconocer que el aprendizaje, en cuanto actividad humana y tarea social es demasiado importante y está profundamente enraizado en el código genético como para ser atrapado y domesticado indefinidamente por la estructura educativa actual. El aprendizaje se liberará pronto del monopolio institucional y cuando 
lo haga, la sociedad tendrá que aprender colectivamente cómo organizarse de manera diferente, cómo desarrollar y cultivar conocimientos y prácticas fuera de las estructuras establecidas, cómo atraer el talento de una nueva generación al empeño educativo, y cómo beneficiar al mayor número posible de personas en la sociedad.

Habiendo dicho lo anterior, pienso que la tutoría es mucho más que un amplio movimiento social a gran escala, producto de una teoría que transforma radicalmente el aprendizaje; también es diseño experimental de aprendizaje futuro en un entorno global en el que las instituciones actuales pierden progresivamente autoridad y control sobre lo que hay que aprender, al tiempo que van siendo desplazadas por formas de organización que apenas estamos aprendiendo a diseñar. La fortaleza mayor de la tutoría está en poder atender poblaciones de niños y adultos a quienes las instituciones actuales no alcanzan a atender bien y están en riesgo de ser objeto de nuevas modalidades de aprendizaje masivas y superficiales, y en ejemplificar una "etapa transitoria" de organización social del aprendizaje, en la que demuestra que es posible abandonar radicalmente formas tradicionales sin dejar de coexistir, aunque alternadamente y de manera simbiótica, con las formas antigüas. También es posible que una de las razones por las que la tutoría ha podido llegar a miles de escuelas es que el sistema educativo en México muestra muchos de los rasgos de una fallida empresa estatal, preocupada por satisfacer sus necesidades internas y solo de manera eventual atenta a su razón de ser. Es urgente que la sociedad más amplia enfrente esta realidad, a pesar de que la estructura actual tenga la capacidad interna, más urgente todavía, de negarla.

Encuentro en la tutoría una poderosa manera de conectar lo micro con lo macro para transformar el aprenizaje en la sociedad. La cultura de la sorpresa, consustancial a la tutoría, es también una manera poderosa de anticipar el futuro del deseño organizacional. Saed Areda, Director Principal de Movilización en NuVu, hace notar que la mayoría de los jóvenes pasa una parte considerable de la vida en entornos educativos en los que son los adultos los que hacen preguntas de las que ellos, los adultos, saben las respuestas (o al menos piensan que las saben) y esperan que los estudiantes las descubran. En vez de esto propone un diseño alternativo para un entorno de aprendizaje en el que tanto adultos como jóvenes emprenden proyectos que a ambos desafían, para los que ninguno tiene la respuesta y por lo mismo deben descubrirla en común, siguiendo un proceso de discusión rigurosa. ¿Por qué? Porque, como varios aprendices en NuVu me han dicho, "adivinar lo que los adultos quieren que digas es aburridísimo y además inútil” Me parece fundamental cultivar deliberadamente la capacidad de sorpresa, tanto en el modelo con el que se aprende, como en el modelo con el que se diseñan los entornos futuros en los que se aprenderá. La sociedad no tiene dificultad para reproducir lo que sabe cómo hacer; pero le resulta difícil imaginar cómo hacer lo que todavía no sabe y aprender 
deliberadamente a hacerlo. Los aprendices en la tutoría, NuVu y HiTech High, y otros que todavía no conocemos, nos están enseñando cómo inventar el futuro. El futuro pide de nosotros cultivar deliberadamente la capacidad de sorpresa.

Esto me lleva de nuevo a la pregunta anterior de cómo podría ayudarnos la tutoría a profundizar la práctica con la que podemos desarrollar y extener esa misma práctica. La respuesta, pienso, está en mantenerse fieles a la teoría de aprendizaje que sustenta la tutoría: aprender rigurosamente a través de preguntas y alentar la práctica deliberada de esperar sorpresas. La respuesta no es elaborar un código de prácticas que puede "implementarse" en diversos entornos; sino promover círculos y redes cada vez más amplias de personas entregadas al aprendizaje en diálogo y que cultivan el interés por la sorpresa. Esto supone necesariamente desarrollar más y más sofisticadas formas de aprendizaje cara a cara, aprovechando la capacidad de adultos y jóvenes que conocen y dominan la práctica.

\section{La tutoría y la neurociencia}

Recientemente, al estar observando dar y recibir tutoría al otro lado de la frontera con México, en el estado de Guanajuato y en las sesiones de capacitación en Chile, pude reconocer con mayor intensidad algo que yo llamo "La mirada" Basta observar el comportamiento casual de adolescentes de I 4 o I 5 años en situaciones sociales. El comportamiento no se diferencia mucho del de cualquier adolescentde de la misma edad: vacilan, coquetean, dramatizan un poco, se juntan, se apartan, etc. Estos comprtamientos son prácticamente los mismos en distintas culturas, y más todavía en ambos lados de la frontera México-Estados Unidos. Pongamos a este adolescente mexicano "normal" en el entorno de aprendizaje propio de la tutoria, en el que se le pide dominar la práctica elemental pero demandante, de aprender en diálogo, mantener la intensidad y el interés del empeño por un buen tiempo (por lo general al menos una hora para un problema o un texto, y a veces más) con el compromiso de que no solo aprenderá el tema, sino que deberá preparar una presentación de lo aprendido y pasar a ser tutor de otro compañero... y entonces ese adolescente "normal" se transforma en uno que tiene "La mirada" Es difícil definir La mirada, pero casi imposible dejar de percibirla: en la calma, en el dominio interior, en la paciencia, la observación aguda, el empeño, el humor; todo esto de una manera que hasta parecería obsesiva. He percibido "La mirada" docenas de veces en entornos radicalmente distintos. La he visto en diversas etapas de desarrollo, entre quienes proceden todavía con incertidumbre y tropiezos y entre quienes se mueven con seguridad y dominio de la práctica. La he visto en adultos y, más importante por su frecuencia, entre los jóvenes. En otras palabras, he visto en ellos, los jóvenes, una disposición hacia el aprendizaje que desafía todos los prejuicios habituales con los que los adultos anticipan el comportamiento de los adolescentes. 
A mípersonalmente, ver con tanta regularidad La mirada entre adolescentes mexicanos en condiciones de pobreza extrema me ha hecho especialmente consciente de la manera tan arraigada con la que la sociedad y las escuelas norteamericanas infantilizan a los adolescentes, al proyectar en ellos teorías más que discutibles del desarrollo que los caracterizan como incapaces de autocontrol, flojos, desorganizados, débiles y ensimismados. Independientemente del valor de las teorías más aceptadas sobre el desarrollo de los adolescentes (en las que cada vez creo menos), los jóvenes que practican la tutoría son absolutamente competentes, muy empeñosos, sumamente atentos, empáticos, maduros y aun carismáticos. Quienes tienen amplia experiencia en la tutoría refieren como algo regular que al principio los jóvenes mexicanos se muestran tímidos y retraídos con los adultos. Después de seguir las prácticas de la tutoría invariablemente se transforman, se vuelven decidores, seguros, sociables y afectuosos. ¿Cómo es que sucede esto?

El acelerado crecimiento orgánico que tiene lugar en el cerebro del adolescente se tensiona entre las emociones, las sensaciones y las reacciones impulsivas -que se localizan hacia la mitad y la parte más baja del cerebro- y el orden, el control y la previsión --que se localizan hacia la parte superior y frontal del cerebro. Mucha gente concibe el desarrollo del adolescente como una mezcla confusa de actos impulsivos, aun irracionales, de los que después, si se superan, los jóvenes se transformarán en seres humanos con suficiente control de sí mismos -siempre ayudados de la sabia guía, la dirección y el control de un adulto bien formado. En otras palabras, si no fuera por el control de los adultos, los adolescentes no madurarían. En esta visión el adolescente común aparece como animal salvaje al que hay que domesticar periódicamente, y el lugar ideal para hacerlo es la escuela. Rara vez, si acaso, caemos en la cuenta de que la tendencia humana inata que impulsa a aprender es potencialmente lo que permite a los adolescentes tener control, visión y orden en sus vidas. El elemento común en los casos que he mencionado -la tutoría, HiTech High y NuVu-es la confianza de los adultos en que asegurando que los adolescentes experimentan el gozo interior de aprender y teniendo la oportunidad de convivir con quienes así aprenden, serán completamente capaces de conducir su desarrollo hacia el orden, la reflexión y el autocontrol. Algo que sorprende sobre manera en estas experiencias es la casi total ausencia de disciplina externa con la que en otras partes se controla el comportamiento, el empeño y la palabra. Otra manera de expresar lo mismo es que el mismo aprendizaje se convierte en poderoso impulsor del desarrollo neurológico cuando exige del aprendiz compromiso intenso y autodirección. De ahí La mirada. Los adolescentes, en estos sitios ejemplares, manifiestan poco o nada de su habitual comportamiento -juguetón, ocurrente y desorganizado- en sus relaciones con los compañeros. Cuando están aprendiendo manifiestan tener propósito firme, creatividad y dominio personal, en mayor grado del que son capaces muchos adultos. 
Es impresionante observar en tiempo real imágenes de un cerebro al momento de aprender: el cerebro "aprende" física y vigorosamente al producir redes cada vez más densas de neuronas, podando literalmente y recomponiendo las que existen, para procesar con más eficiencia conocimientos y sentimientos en nuevas estructuras. Durante la adolescencia estos procesos llegan al máximo. En los primeros años de la adolescencia la masa cerebral crece al límite y empieza a declinar conforme se consolida y reorganiza, al empezar la edad adulta. ¿Cómo se produce este desarrollo y de qué depende el modo como ocurre? Se han dado diversas respuestas y se conjeturan otras que habrá que explorar; pero hay una respuesta para la que existe suficiente evidencia: el cerebro "aprende" en este proceso de crecimiento y consolidación a través del habla, el medio por el que el mundo se hace inteligible. Hablar y hacer sentido de las cosas crean literalmente y afirman las estructuras que se usarán después para pensar y crear.

En el trabajo de observación de salones de clase que he venido haciendo hace más de doce años, anotaba regularmente el tiempo que hablaban los jóvenes y el que hablaban los adultos. En los salones que observé en escuelas norteamericanas encontré un patrón bastante estable de 85-90\% de habla de adultos y 10-15\% de habla de estudiantes. Los adultos en general hablaban en largos y ampulosos períodos, frecuentemente con frases o párrafos incompletos. Los estudiantes, por lo general, hablaban brevemente, emisiones de menos de doce palabras; también en fases incompletas, frecuentemente para terminar con dos o tres palabras la frase que habían iniciado los adultos. Si la teoría del desarrollo del cerebro considera que hablar produce aprendizaje, lo que yo observé sugiere que las oportunidades de desarrollo mental habían sido mínimas. En estas circunstancias, ihacia dónde se orientará la urgencia humana de producir lenguaje y generar crecimiento? Pienso que la producción de lenguaje tendrá lugar entre compañeros y, posiblemente también al interactuar con adultos en entornos no escolares, lo que equivale a decir que estamos obligando a los jóvenes a pasar gran parte de sus vidas en entornos que contribuyen poco, si acaso, al desarrollo de sus funciones neurocognitivas.

Lo que me sorprende en la tutoría es la extraordinaria abundancia de conversación seria por parte del tutor, sea este joven o adulto. La diferencia entre esta manera de aprender y la de escuelas convencionales es tan amplia, tan diferente, que mis habituales prácticas de observación se hacen innecesarias. La práctica más sorprendente es la simple cantidad de plática que induce la tutoría, con preguntas y respuestas bien estructuradas, así como referencias precisas a textos y problemas. También sorprende la profundidad del diálogo. Lo que en una clase convencional se reduciría a solo una pregunta y su respuesta, en la tutoría se convierte en series de preguntas desafiantes, de compejidad creciente, que se alternan al estar razonando sobre un problema matemático o al discutir sobre el significado de una frase, una 
estrofa, un párrafo o un texto más amplio. Personalmente puedo decir que nunca me sentí tan incómodo y tan desafiado intelectualmente como cuando recibí tutoría de dos jovencitas de catorce años sobre geometría y poesía histórica; acabé como si mi mente hubiera estado durante dos horas ejercitándose en un gimnasio.

En HiTech High y NuVu se observa una producción verbal semejante. Ahí los adultos muy rara vez, si acaso, responden directamente a las pregunas que les hacen los estudiantes; les preguntan más bien de dónde vienen sus preguntas y hacen otras más con el objeto de ayudarlos a clarificar lo que piensan y lo que quieren encontrar. En NuVu, los intercambios verbales, al principio, deben parecer muy amenazantes a quienes llegan acostumbrados a la falsa cortesía de la cultura escolar tradicional. Cada etapa del diseño concluye con una discusión de todo el grupo en la que cada participante (habitualmente $40 \circ 50$ estudiantes y cuatro o cinco adultos) hace preguntas, cuestiona aspectos del diseño y propone alternativas para algún prototipo o para el diseño final. Es normal que en las sesiones de crítica se descarten sin más ideas de diseño ya discutidas y elaboradas, y que los equipos propongan empezar de nuevo a partir de otra idea. Saeed Areeda, uno de los fundadores de NuVu dice que los estudiantes llegan de la escuela al estudio pensando que pueden responder con facilidad a los desafíos que presenta un diseño, y al principio se resisten y enojan con las críticas a sus ideas. Saeed explica que en la escuela se habitúan a pensar que ser talentoso equivale a pensar rápido y fácil, y se sorprenden cuando la crítica los obliga a profundizar más lo que piensan y proponen. Conforme aprenden la disciplina propia de los estudios de diseño arquitectónico, participan más activamente en la crítica y manejan con mayor soltura el lenguaje profesional de un proceso de diseño.

Hay muchas avenidas de desarrollo para un adolescente, pero verbalizar es ciertamente una de las más importantes. El lenguaje ofrece múltiples y diversas oportunidades de desarrollo Los aprendices en la tutoría, por ejemplo, desarrollan un amplio vocabulario con el que expresan empatía y ayudan a sus compañeros. El simple hecho de permanecer uno frente al otro durante un buen tiempo hace necesariamente que jóvenes o adultos generen diálogos de gran complejidad y muy variados. Una y otra vez he visto a tutores jóvenes que ensayan distintas preguntas para lograr descubrir lo que impide a sus aprendices avanzar en la comprensión de un problema o un texto. He visto con admiración a dos chiquillas de catorce años, enfrascadas en un diálogo durante hora y media desentrañando el sentido, línea por línea, de un difícil texto original de historia de México. La tutora demostró gran habilidad para ayudar a su aprendiz a encontrar sentido del contexto y relacionaba constantemente el marco general de la historia con los detalles del texto. Cuando terminó la tutoría les pregunté qué pensaban hacer, y la tutora respondió que ahora su aprendiz le daría tutoría en matemáticas. 
Finalmente, la tutoría tiene mucho qué enseñarnos sobre la neurobiología del estrés y el aprendizaje. Es difícil pensar en un entorno neurobiológicamente más adverso al aprendizaje que una escuela regular norteamericana. Invariablemente se juzga a estudiantes que vienen de contextos sociales muy diversos, y tienen aptitudes y actitudes diferentes, conforme a comportamientos y atributos que arbitrariamente se espera tendrá un grupo según la edad y el grado. El tiempo se segmenta en pequeños tramos y lo que en ellos hay que hacer se decide sin tener en cuenta si la tarea que se pide corresponde al grado de madurez de cada individuo. Los estudiantes tienen pocas oportunidades de escoger aprender lo que les interesa y aun se les dice oficial y continuamente que sus intereses deben subordinarse a lo que pide la autoridad externa. En este ambiente los adultos de manera casi sistemática parecen programados para ignorar y hasta exacerbar las tensiones que el entorno produce en los estudiante. Y más importante todavía, se piensa que un alto nivel de estrés corresponde al rigor de buen aprendizaje, especialmente tratándose de adolescentes. Una escuela "difícil" es una "verdadera escuela". Se cree que aprender a vivir con altos niveles de estrés es buena preparación para el mundo adulto.

El cerebro y el cuerpo manejan el estrés por medio de neurotransmisores, que son básicamente formas diversas de sustancias químicas naturales y hormonas, que activan áreas particulares del cerebro. El área que responde al estrés es la amígdala, en la mitad inferior del cerebro, y regula las dos conocidas respuestas de agresión o de huída. De hecho las respuestas al estrés, como las funciones más relevantes del cerebro, involucran muchas más áreas; gracias a la investigación aprendemos más cada día del modo como actúa el estrés. Lo que es más importante en las respuestas al estrés es que se disparan, en parte, por alguna amenaza; pero estas respuestas, al quedar asociadas a un determinado entorno o situación, literalmente reprograman la respuesta de la persona al asociar sentimiento con memoria. En situaciones muy estresantes las funciones superiores se suspenden y el cerebro sólo busca la sobrevivencia. Enfrentar con frecuencia situaciones estresantes lleva a huír y adoptar instintivamente comportamientos defensivos. Las situaciones estresantes producen recuerdos que después se activan en situaciones semejantes; el cuarto, la iluminación, el olor, la voz pueden iniciar respuestas de estrés sin que necesariamente estén presentes acciones generadoras de estrés. Mantener normalmente a los estudiantes en situaciones de estrés en un mismo salón casi garantiza que con el tiempo asociarán ese espacio con reacciones de estrés y encontrarán maneras de alejarse de ese entorno y de evitar las actividades que primero les generaron el estrés.

Un descubrimiento sorprendente de la neurociencia -que me incomodó y me pareció fuera de razón cuando lo leí por primera vez- es que el cerebro responde neuroquímicamente al aburrimiento de manera casi idéntica a la manera como 
responde ante una amenaza. Esto quiere decir que manteniendo a una persona en un entorno en el que regularmente habrá pocos estímulos, además de desinterés afectivo e intelectual, tendremos la misma respuesta de huída y rechazo que se da ante el peligro externo. Baste esto para subrayar que en situaciones de aburrimiento el cerebro suspende funciones biológicas normales. De hecho el aburrimiento activa y estimula la amígdala para liberar las mismas hormonas que generan respuestas de fuga o de agresión.

En mis observaciones en salones de clase de escuelas americanas registraba regularmente el lenguaje corporal, las miradas y expresiones verbales que revelan interés en el que aprende. Mis colegas y yo pedíamos regularmente a algunos estudiantes que nos dijeran qué era lo que estaban aprendiendo en algún momento particular de la clase. De esas observaciones surgió lo que he dado en llamar "la regla de tercios". En las mejores circunstancias, que no eran las más comunes, un tercio de la clase parecía no tener interés alguno (vista perdida, cuerpo suelto), otro tercio parecía formalmente interesado (mostraban atención, pero no en sintonía con lo que hacía y decía el maestro) y el otro tercio estaba realmente interesado (mirándose y viendo al maestro). Las manifestaciones de interés en algunos estudiantes variaban, pero la proporción de tercios se mantenía relativamente constante. Aunado a este comportamiento se daba otro más desolador. Cuando preguntábamos a los estudiantes por lo que estaban estudiando, estuvieran o no empeñados en el estudio, sus respuestas caían en dos categorías: simplemente repetían la instrucción del maestro (no nos decían lo que estaban aprendiendo) o nos decían con sencillez que no sabían lo que estaban aprendiendo. Como sucede con esos patrones de comportamiento, la variabilidad en muchas escuelas iba de un extremo a otro -en algunos salones de clase hervía el entusiasmo, la comunicación era intensa, todo mundo estaba comprometido con el aprendizaje; mientras que otros salones eran entornos completamente apagados, con pocos intercambios verbales en el contínuo enseñanza-aprendizaje. En escuelas de adolescentes con frecuencia teníamos que registrar el número (no despreciable) de alumnos que se quedaban dormidos en algún momento de la clase.

En los Estados Unidos las llamadas "escuelals sin excusas" tienen protocolos escritos para inducir atención, contacto visual y responder preguntas de los maestros, con lo que crean un entorno sumiso de trabajo escolar. Sería interesante saber si en estas escuelas los estudiantes responden de la misma manera a la pregunta "¿Que están aprendiendo"? Se puede decir de estas rutinas externas que inducen estrés de manera sistemática a fin de estimular y mantener un entorno de aprendizaje centrado en el adulto. En estos salones a los alumnos rebeldes se les señala y se les pide que abandonen el salón o que se sienten dando la espalda. Aunque es casi imposible, resultaría interesante hacer escaneos cerebrales para medir reacciones al estrés en 
estos entornos y compararlo con las reacciones en otros entornos de aprendizaje. Añadamos esta idea a la lista de experimentos interesantes en neurociencia que jamás se harán. Sin embargo experimentos semejantes en entornos bien controlados muestran impactos considerables en la memoria activa y los procesos cognitivos en lugares donde la conducta de los participantes se norma regularmente por órdenes y señales externas.

Algo notable en la tutoría es la casi total ausencia de prácticas que inducen estrés. Cada estudiante escoge la tarea de aprendizaje que le interesa, en ocasiones apoyado por un adulto, en otras por un compañero, pero siempre se respeta lo que el estudiante quiere aprender. La práctica tutora emplea la empatía, el propósito preciso, el empeño persistente como fuentes de motivación; en el supuesto de que toda persona al tener la oportunidad en un entorno convivial, activará necesariamente su deseo innato de aprender. Todavía de manera más enriquecedora, durante la tutoría se le pide frecuentemente al aprendiz expresar lo que piensa haber aprendido, a fin de poder emplearlo como peldaño para el siguiente nivel de conocimiento. Las presentaciones que demuestran la competencia del aprendiz para pasar a ser tutor en un tema particular inducen naturalmente ansiedad por ser públicas. Pero esas presentaciones vienen precedidas de abundante práctica y ejercicio continuo que dan seguridad.

En HiTech High y NuVu se observan las mismas prácticas. Los estudiantes asumen el autocontrol al participar regularmente en trabajos de grupo en los que el diálogo crítico as lo normal. Los estudiantes que llegan de escuelas "regulares", acostumbrados a entornos en los que vale más la pasividad y el sometimiento que la discusión crítica, se angustian al principio, pero pronto se adaptan a un medio en el que la crítica es la base del aprendizaje. Más importante todavía, a esos estudiantes se les ayuda a adoptar un lenguaje en el que la crítica se hace con empatía y respeto hacia los compañeros. El lema central en NuVu, por ejemplo, es "critica la idea o el prototipo, no la persona". La idea central del trabajo es que la persona o el equipo aproveche la crítica para producir un mejor diseño, no para salir descorazonados, pensando que si ahora fallaron, también van a fallar en el siguiente diseño.

Este largo excurso sobre la neurociencia y el aprendizaje se explica por la necesidad que tenemos de saber crear y propagar entornos que efectivamente sostengan el aprendizaje. Entre más observo entornos que responden explícitamente a bien fundadas teorías del aprendizje, aun cuando entre ellas haya diferencias, más admiro y respeto el complejo recipiente humano en el que queremos verter las ideas y expectativas de lo que consideramos "bueno". Me ha sorprendido que las teorías más prometedoras en el empeño de diseñar nuevos entornos de aprendizaje son aquellas que más respetan a las personas, organismos que están biológica y genéticamente hechos para aprender; teorías que aceptan el principio de buscar 
deliberadamente sorpresas, y teorías que desafían el monopolio que las instituciones establecidas pretenden tener sobre el discurso, las creencias y las reglas del aprendizaje como actividad individual y social. Mi experiencia reciente me hace sumamente escéptico sobre el futuro de la escuela y enormemente optimista sobre el futuro del aprendizaje. Agradezco a los extraordinarios adolescentes, especialmente a Maricruz y a Scarlet, que me enseñaron matemáticas y poesía, tan bien y tan profundamente, que lograron cambiar mi percepción de lo que soy como aprendiz. 